\title{
Evaluación de la carga académica de una carrera de ingeniería según el modelo basado en competências
}

\section{Evaluation of the academic load of an engineering degree program according to the competency-based model}

DOI: $10.46932 / \operatorname{sfjdv3n1-031}$

Received in: Dec 30st, 2021

Accepted in: Jan 1th, 2022

\author{
Myriam Raquel Gaete Gaete \\ Master in Operation Managment \\ CEIA Victoria talca \\ Niches Km. 1, Curicó, Chile. \\ E-mail: Myriam.gaete@utalca.cl \\ Daniela Cecilia Sánchez Oyarzún \\ English teacher \\ University of Talca \\ 7 y 8 oriente 2 sur, 1311, Talca, Chile \\ E-mail: Sanchezdaniela2014@gmail.com
}

\section{RESUMEN}

En la actualidad las universidades chilenas adscritas al CRUCH definen la carga efectiva del estudiante y docente según el Sistema de Créditos Transferibles SCT-Chile. Este sistema a pesar de ser un marco regulatorio a nivel nacional que permite la movilidad estudiantil entre otros beneficios, a la fecha no existe otro tipo de herramientas que corroboren las estimaciones realizadas. Por tanto, la principal tarea de esta investigación surge a partir de la necesidad de cuantificar la carga académica de una carrera de ingeniería a través de un modelo matemático, considerando que esta se encuentra inserta en el modelo de educación basado en competencias. El modelo matemático se centra en la valorización de cada uno de los saberes que componen la trayectoria de aprendizaje según la taxonomía del saber, el contenido y el factor de desarrollo de la competencia con el propósito de corroborar las estimaciones declaradas en la trayectoria de aprendizaje según el SCT-Chile. Para esto se cuantifico la carga académica por semestre, competencias y módulos de la trayectoria de aprendizajes de una carrera de Ingeniería de una universidad chilena. Los resultados muestran que se presentan desequilibrios entre la cantidad de horas de declaradas según el Sistema de SCT-Chile y la carga académica estimada a partir del modelo matemático.

Palabras claves: Metodología cuantitativa, evaluación de programas por competencias, caso de estudio ingeniería.

\begin{abstract}
At present, Chilean universities affiliated to the $\mathrm{CRUCH}$ define the effective student and faculty load according to the Transferable Credit System SCT-Chile. Although this system is a national regulatory framework that allows student mobility among other benefits, to date there are no other tools to corroborate the estimates made. Therefore, the main task of this research arises from the need to quantify the academic load of an engineering career through a mathematical model, considering that it is inserted in the competency-based education model. The mathematical model focuses on the valuation of each of the knowledge that make up the learning path according to the taxonomy of knowledge, the content and
\end{abstract}


the competence development factor in order to corroborate the estimates stated in the learning path according to the SCT-Chile. For this purpose, the academic load per semester, competencies and modules of the learning trajectory of an engineering degree of a Chilean university were quantified. The results show that there are imbalances between the number of hours declared according to the SCT-Chile System and the academic load estimated from the mathematical model.

Keywords: Quantitative methodology, competency-based program evaluation, engineering case study.

\section{INTRODUCCIÓN}

A partir del fenómeno conocido como la globalización, los centros educativos superiores han debido de efectuar un plan estratégico de formación para profesionales integrales, por lo cual se ha debido de establecer una reformulación en cuanto al modelo tradicional adoptando uno que propicie los saberes educativos: saber ser, saber hacer y saber conocer (Perez, 2012).El modelo adoptado ya hace unos años en las aulas chilenas de educación superior no es más que el modelo por competencias el cual sustenta la articulación de la teoría y la práctica.(Muñoz, 2006)

Es por este motivo que, desde la perspectiva de innovación curricular, surge la educación basada en competencias. Este modelo se presenta como un modelo flexible el cual prioriza las ideas de eficiencia, calidad y competitividad expuestos en los modelos de certificación y evaluación de la calidad educativa y profesional. Por otra parte, hace referencia a la búsqueda de la homologación que debe de presentarse entre los planes de estudios de diversas instituciones a lo largo del mundo (Díaz Barriga, 2006).

Sin embargo, dentro del enfoque basado en competencias no se ha diseñado la forma de medir cuantitativamente su éxito o fracaso dentro de las aulas chilenas; es por este motivo, que el presente artículo genera una cuantificación de la calidad del modelo educativo establecido por las instituciones con el fin de identificar si realmente este logra satisfacer las necesidades de la educación chilena. Para tal fin, se tomó como caso de estudio la trayectoria de aprendizaje de una carreara de ingeniería de una universidad chilena.

\subsection{REVISIÓN BIBLIOGRÁFICA}

El modelo de educación basada en competencias se presenta hoy en día como una opción más fresca para la construcción del diseño curricular y por ende para el desarrollo del proceso de aprendizajeenseñanza. El modelo en si con el transcurso de los años ha ido adoptado diferentes definiciones según los diversos autores. Por una parte, Tardiff define las competencias como:

"un saber actuar complejo que se apoya en la movilización y la combinación eficaz de una variedad de recursos internos y externos dentro de una familia de situaciones Tardiff (2006). 
Las competencias llegan a los planes de estudios a través del diseño curricular. El diseño curricular es la llave maestra que conecta a la institución, las competencias y el perfil de egreso con la trayectoria de aprendizaje, los docentes y el estudiante.

El diseño curricular es un proceso importante dentro de la creación de un programa por competencias, puesto que alude principalmente a organizar un plan educativo a través de la estructuración de las necesidades de los estudiantes es por esto que para el cumplimiento de las competencias es necesario un diseño curricular que permita que el estudiante sea capaz de desarrollar las mismas según la intencionalidad que le entregue el diseño curricular.

Siguiendo con este razonamiento, Pérez (2011), menciona a el diseño curricular como una dimensión del currículum integradora de la concepción de lo educativo, como fenómeno, de las metas o fines de la acción del proceso de enseñanza-aprendizaje y de los los recursos complementarios necesarios para lograr los resultados esperados, manteniendo una estructura y un orden.

A partir del diseño curricular, se forma el sistema de créditos transferibles utilizados en las universidades de la CRUCH, este se desglosa en tres fases que permiten orientar la implementación de las diversas instituciones. La primera de ellas hace alusión a la carga de trabajo total que deben mantener los estudiantes por lo que considera el tiempo invertido por cada una de las actividades que el estudiante presenta (salidas a terreno, redacción de informes, talleres, cátedras directas etc.) en segunda instanciase hace énfasis al tiempo total que el estudiante dedica a su carrera por lo que se estima un trabajo semanal de 45 a 50 horas considerando tiempo completo de una carrera. Como última fase se encuentra el componente normalizador anual el cual asigna un número de créditos específicos a las diversas actividades curriculares a lo largo de un año académico de un plan de estudio.

De este modo y a partir del diseño curricular, nace el sistema de créditos transferibles de las universidades de la CRUCH (en Chile). La implementación del sistema se desagrega en tres componentes y seis principios que entregan una orientación general. El primer componente señala que el sistema se centra en la carga de trabajo total de los estudiantes. Lo que quiere decir, que se analiza cada una de las actividades que debe realizar el alumno para el logro de los objetivos de aprendizaje de cada uno de los módulos, considerando clases presenciales, evaluaciones trabajo autónomo y actividades practicas entre otras. El segundo componente habla del tiempo que el estudiante dedica semanalmente a su carrera. Se asume como máximo una carga de trabajo semanal de 45 a 50 horas para una dedicación de tiempo completo en una carrera de pregrado.

Y el tercer componente es la asignación anual de un número específico de créditos a las distintas actividades curriculares de un año cualquiera del plan de estudios. Se ha convenido que a la carga de trabajo anual debe estar entre 1.440 y1.900 horas, a lo que se le asocian 60 créditos SCT. 
En este sentido, el objetivo de este estudio es establecer un modelo matemático que permite revisión y valorización de la carga de trabajo anual de los estudiantes con el propósito de contribuir de manera cuantitativa a la medición de las competencias en una carrera de ingeniería. Para esto se parte definiendo el modelo de valorización y luego se realiza un estudio de caso para verificar la metodología propuesta.

\section{MÉTODO}

La metodología de evaluación del diseño curricular que se propone se caracteriza por ser un método hibrido, donde se combina lo cualitativo con los cuantitativo. El método se divide en las siguientes cuatro etapas, donde las dos primeras corresponden a un análisis cualitativo del perfil de egreso, competencias y saberes del programa de estudio, seguidos de una tercera y cuarta etapa cualitativa, donde se evalúa la carga académica a través de un modelo matemático que permite la valorización de los saberes:

\section{- $\quad$ Análisis del perfil de egreso y las competencias}

En cuanto a esta parte del proceso en cuestión se comenzó primero que todo con el análisis del perfil de egreso y las competencias que posee por lo que la idea central fue identificar si las competencias apuntaban directamente con lo que el perfil de egreso estipula. Para esto se cruzó la información del perfil de egreso con las competencias, buscando concordancia entre ambas.

\section{- $\quad$ Análisis de la trayectoria de aprendizaje y competencias}

Siguiendo con la metodología de desarrollo de un diseño curricular de un módulo, se prosiguió con el análisis de la trayectoria de aprendizaje y sus competencias, para esto en primeria instancia se realizó la revisión de los saberes etapa que consiste en identificar si la redacción y síntesis de los mismos corresponde a lo que se estipula al momento de la planificación siguiendo los lineamientos tales como Explicar el sentido del módulo.

\section{- Modelo de evaluación del sistema de créditos de la carrera}

Durante esta etapa del proceso se valoriza cada una de las competencias, en relación con la cantidad de horas presenciales y de trabajo autónomo que tiene el estudiante, con el propósito de verificar el nivel de logro propuesto para la competencia y la carga académica del estudiante. En nuestra opinión el total de créditos por competencia se define como la multiplicación de tres factores: la jerarquía de objetivos de aprendizaje (LOHk), el factor de desarrollo de la competencia esperado en los módulos que tributan a la misma (CDFi) y la profundidad del contenido asociada al saber (CDk). 


$$
T C c_{j}=\sum_{i=1, k=1}^{n, l} L O H_{k} * C D F_{i, j} * C D_{k} \quad \forall j \in\{1 \ldots m\}
$$

Donde TCcj es el total de créditos de la competencia j (Total credits for competition), LOHk la jerarquía en la taxonomía del saber k (Learning Objectives Hierarchy), CDFi,i el factor de desarrollo de la competencia esperado en el módulo i(Competition development factor), CDk corresponde a la profundidad del contenido en el saber k (Content depth), n es el número de módulos con los que cuenta la carrera, m es la cantidad de competencias de la carrera y l es la cantidad de saberes con los que cuenta la trayectoria de aprendizaje.

Para complementar esta definición, a continuación, se explican en detalle cada uno de estos parámetros: Jerarquía de los objetivos de aprendizaje (Learning Objectives Hierarchy, LOHk): En primer lugar, debemos jerarquizar los saberes en niveles de complejidad, para esto podemos usar modelos como las taxonomías de objetivos de aprendizaje. Estos niveles deben ser categorizados en orden creciente de complejidad; A modo de ejemplo, se podría definir como base la taxonomía de Bloom-Anderson considerando seis niveles: Recordar, comprender, aplicar, analizar, evaluar y crear. Cada uno de estas categorías se valoriza en relación a un factor que varía entre 1 y 12 . Siendo 1 el nivel más bajo de la Jerarquía y 12 el más alto; Factor de desarrollo de la competencia (Competition development factor, $\mathrm{CDFi}, \mathrm{j}$ ): Corresponde a una traducción numérica del nivel de dominio con el que el estudiante llega al módulo respecto a la competencia. Se sugiere que ese valor varía entre 1 y 3 , siendo 1 el nivel de dominio máximo que es adquirido por el estudiante antes de llegar a esta competencia el cual es bajo y 3 cuando el nivel es alto; Profundidad del Contenido (Content Depth, CDk): se define como el nivel de complejidad del contenido considerando como línea base los conocimientos adquiridos hasta el nivel inferior. Por ejemplo, al analizar la trayectoria de aprendizaje de la educación media, la línea base corresponde a todos los saberes adquiridos en la educación básica. Estos niveles deben estar compuestos por tres o más jerarquías que son valorizadas en una escala de 1 a 3.

Luego el análisis de la trayectoria de aprendizaje completa se realiza en base a la siguiente formula, donde TDT es el total del crédito de la carrera (Total degree credits).

$$
T D T=\sum_{i=1}^{n} T C c_{j}
$$

Mientras que la evaluación de los módulos se realiza con la siguiente formula, donde TMCi (Total module credits) es el total de créditos del módulo i: 


$$
P C_{k} \quad T M C_{i}=\sum_{k=1}^{l} L O H_{k} * F D C_{i, j} *
$$

- Comparación con créditos declarados en la trayectoria de aprendizaje

En la presente etapa se comienza con el análisis de los resultados desde una perspectiva cuantitativa por módulos, competencias y semestre. Por módulos se propone segregar los módulos según la siguiente categorización de los resultados; Módulo con exceso de carga: hace referencia a todo modulo que cuenta con a lo menos un $10 \%$ más de horas valorizadas respecto a la cantidad de horas declaradas según el sistema de créditos; Módulo con déficit de carga académica: se refiere a todo modulo que cuenta con a lo menos $10 \%$ menos de horas valorizadas respecto a la cantidad de horas declaradas según el sistema de créditos; Módulos ajustados: hace referencia a todo modulo que cuenta con una cantidad de horas valorizadas entre un $90 \%$ y $110 \%$ respecto a la cantidad de horas declaradas según el sistema de créditos

Una vez realizado el análisis comparación por modulo se valorizan las competencias según el nivel de logro; como resultado, se espera que a mayor nivel de logro mayor sea la carga académica estimada.

Finalmente se evalúa la carga académica declarada en la trayectoria de aprendizaje de cada uno de los semestres del programa analizado, esperando que la carga académica estimada con el modelo se asemeje lo más posible a lo establecido. La Tabla 4 muestra el formato del resumen del semestre, la carga académica estimada, la carga real y la diferencia entre ambos.

\section{RESULTADOS}

\subsection{UNIDAD DE ANÁLISIS}

En este trabajo se analizó una carrera de ingeniería en una universidad de Chile. Dicha carrera cuenta con un total de 11 semestres distribuidos en 6 años de duración, 330 SCT-Chile y con un total de 8.370 horas declaradas.

\subsection{ANÁLISIS DEL PERFIL DE EGRESO Y LAS COMPETENCIAS}

A continuación, se presenta una tabla se expositiva la cual evidencia las competencias y el lugar situado al que hacen relación con el perfil de egreso establecido por la carrera en particular. 
Tabla 1. Análisis del perfil de egreso de una carrera de ingeniera

\begin{tabular}{|c|c|c|}
\hline Competencias & $\begin{array}{l}\text { Nivel de } \\
\text { logro }\end{array}$ & Perfil de egreso \\
\hline Competencia 1 & Avanzado & \multirow{4}{*}{$\begin{array}{l}\text { El perfil de egreso de la carrera expone de manera concreta estas } \\
\text { competencias por lo que al momento de evidenciarlas no es difícil } \\
\text { encontrarlas dentro del mismo, de esta forma se le otorga a la carrera } \\
\text { la plusvalía que la misma estipula a los futuros estudiantes egresados. }\end{array}$} \\
\hline Competencia 2 & Intermedio & \\
\hline Competencia 3 & Avanzado & \\
\hline Competencia 4 & Intermedio & \\
\hline Competencia 5 & Avanzado & $\begin{array}{l}\text { La competencia } 5 \text {, no se encuentra declarada dentro del perfil de } \\
\text { egreso de la carrera por lo que, no es posible triangular si la } \\
\text { competencia realmente incide o no dentro de la formación del } \\
\text { profesional al que el perfil de la carrera apunta. }\end{array}$ \\
\hline Competencia 6 & Avanzado & \multirow{7}{*}{$\begin{array}{l}\text { Las competencias se encuentran declarada dentro del perfil de egreso } \\
\text { de la carrera, por lo que es posible evidenciar su incidencia dentro de } \\
\text { la misma para que así los futuros alumnos y egresados puedan } \\
\text { identificarla. }\end{array}$} \\
\hline Competencia 7 & Intermedio & \\
\hline Competencia 8 & Intermedio & \\
\hline Competencia 9 & Avanzado & \\
\hline Competencia 10 & Avanzado & \\
\hline Competencia 11 & Intermedio & \\
\hline Competencia 12 & Intermedio & \\
\hline
\end{tabular}

A través del análisis efectuado entre las competencias y el lugar en que se encuentran en el perfil de egreso, se establece como conclusión que de las doce competencias establecidas en el perfil de egreso de la carrera; solo once se encontraban situadas en el mismo, dejando de la lado solo una, por lo cual se propone que como una mejora considerable, se realice la modificación del perfil de egreso de manera tal que integre dicha competencia permitiendo así una coherencia entre lo establecido por la carrera y las competencias de la misma.

\subsection{ANÁLISIS DE LA TRAYECTORIA DE SABERES Y COMPETENCIAS}

Continuando con el trabajo de análisis; se dispuso en esta parte de la investigación, a analizar los saberes que poseía la carrera de ingeniería de una universidad de chile, por lo que se pudo identificar la existencia de muchos saberes con problemas de redacción y síntesis, por lo que se decidió restructúralos para cumplir con las exigencias que demanda el perfil de egreso. La presente tabla se utilizó con la finalidad de entregar la información de la nueva propuesta de reestructuración de los saberes en contraste a los ya existentes.

Tabla 2. Ejemplo del análisis de los saberes de una competencia de ingeniería

\begin{tabular}{|l|l|c|}
\hline & Saberes del plan de estudios & Cambio \\
\hline & Diseñar la estructura general del Sistema de Operaciones. & Redacción \\
\hline & Balancear la capacidad de procesos a través de simulación & Redacción y agrupación \\
& Balancear la capacidad de procesos & Síntesis \\
\hline & $\begin{array}{l}\text { Levantar y analizar el estado del arte de procesos y procedimientos en } \\
\text { sistemas de operaciones de bienes y servicios. }\end{array}$ & \\
\hline
\end{tabular}

Una vez realizado el análisis de la trayectoria de saberes y competencias se pudo identificar que en gran parte los saberes poseían errores de escritura, síntesis o una mala organización por lo cual se 
decidió la reagrupación de algunos con la finalidad de mejorar estos mismo y así generar un impacto dentro de la trayectoria de la carrera.

\subsection{EVALUACIÓN DEL SISTEMA DE CRÉDITOS DE LA CARRERA}

Siguiendo la metodología propuesta en la sección anterior, en la etapa uno del algoritmo se valorizo cada uno de los saberes presentados, obteniendo como resultado una valorización total de 8.594,59 horas equivalentes a 310 créditos (se excluyen los 20 créditos de los electivos). Según el sistema de créditos transferibles utilizado por la universidad deberían ser 8.370 horas.

En cuanto a la evaluación de los módulos se consideró para la evaluación la siguiente tabla con la valorización en horas lectivas y de trabajo autónomo según la LOHk la jerarquía en la taxonomía Objetivos de Aprendizaje del saber k:

Tabla 3. Valorización de los saberes según su nivel en la Taxonomía de Bloom

\begin{tabular}{|l|c|c|r|}
\hline & Horas trabajo autónomo & Horas trabajo presencial & Total Hr \\
\hline Crear & 2,67 & 1,33 & 4,00 \\
\hline Evaluar & 2,33 & 1,00 & 3,33 \\
\hline Analizar & 2,00 & 1,00 & 3,00 \\
\hline Aplicar & 1,67 & 1,00 & 2,67 \\
\hline Comprender & 1,33 & 0,67 & 2,00 \\
\hline Recordar & 1,00 & 0,67 & 1,67 \\
\hline Reconocer & 1,00 & 0,67 & 1,67 \\
\hline
\end{tabular}

Obteniendo que la carrera cuenta con 882 saberes distribuidos en los seis niveles de la taxonomía de aprendizaje usada (taxonomía de Bloom), donde la mayoría de los saberes se presentan en el nivel aplicar.

Tabla 4. Evaluación de los saberes según su nivel en la Taxonomía de Bloom

\begin{tabular}{|c|c|c|}
\hline Taxonomía de Bloom & Cantidad de saberes & Porcentaje de saberes \\
\hline Crear & 87 & $10 \%$ \\
\hline Evaluar & 73 & $8 \%$ \\
\hline Analizar & 107 & $12 \%$ \\
\hline Aplicar & 310 & $35 \%$ \\
\hline Comprender & 124 & $14 \%$ \\
\hline Recordar & 166 & $19 \%$ \\
\hline Reconocer & 15 & $2 \%$ \\
\hline Total & $\mathbf{8 8 2}$ & $\mathbf{1 0 0 \%}$ \\
\hline
\end{tabular}

Y el $\mathrm{CDF}_{\mathrm{i}}$, el factor de desarrollo de la competencia esperado en el módulo i se detalla en la siguiente tabla, donde los primeros semestres tienen asignado un valor más alto, considerando que el estudiante a medida que avanza en la carrera requiere de menos horas de trabajo para obtener un mismo resultado: 
Tabla 5. Valorización de los saberes según la profundidad del contenido

\begin{tabular}{|c|c|}
\hline $\boldsymbol{C D}_{\boldsymbol{i}}$ & Semestre del módulo \\
\hline 3 & $1-2$ \\
\hline 2.5 & $3-4$ \\
\hline 2 & $5-6$ \\
\hline 1.5 & $7-8$ \\
\hline 1 & $9-10-11$ \\
\hline
\end{tabular}

$\mathrm{CD}_{\mathrm{k}}$ corresponde a la profundidad del contenido en el saber $\mathrm{k}$ es un factor que varía entre 1 y 3 de 0,5 en 0,5. Según el análisis de expertos de la carrera de los 882 saberes se obtuvo que la mayoría de los saberes se encuentra con un factor de desarrollo superior a 2, lo que significa que la mayoría de los contenidos entregados en la carrera son de un nivel medio y alto.

Tabla 6. Evaluación de los saberes según la profundidad del contenido

\begin{tabular}{|l|l|l|}
\hline & Cantidad de Saberes & Porcentaje \\
\hline 1,0 & 136 & $15 \%$ \\
\hline 1,5 & 202 & $23 \%$ \\
\hline 2,0 & 120 & $14 \%$ \\
\hline 2,5 & 212 & $24 \%$ \\
\hline 3,0 & 212 & $24 \%$ \\
\hline Total & $\mathbf{8 8 2}$ & $\mathbf{1 0 0 \%}$ \\
\hline
\end{tabular}

\subsubsection{Comparación con créditos declarados en la trayectoria de aprendizaje}

\subsubsection{Comparación por módulo.}

Posteriormente, al evaluar los módulos según el modelo de valorización propuesto, se obtiene que solo un $37 \%$ de los módulos estudiados son módulos ajustados; lo que significa que prácticamente no existe diferencia entre las horas declaradas en la trayectoria de aprendizaje y los resultados obtenidos mediante la valorización de los saberes; por otra parte, un 32\% de los módulos presenta un déficit entre la carga académica declarada y la valorización, lo que significa que los estudiantes requieren de menos horas de estudio para adquirir las competencias declaradas en esos módulos. Y por último un 31\% de los módulos de la carrera estudiada presentan un exceso de carga académica, lo que se traduce en que los estudiantes requieren de un mayor número de horas de carga académica para cumplir con los requerimientos del módulo.

Tabla 7. Resultados del análisis realizado por módulos

\begin{tabular}{|l|l|l|l|l|}
\hline & Cantidad & $\boldsymbol{T M C}_{\boldsymbol{i}}$ & SCT-Chile & Diferencia \\
\hline Módulos ajustados & 23 & $2.888,50$ & $2.889,00$ & $-0,5$ \\
\hline Módulos con déficit de saberes & 20 & $1.457,50$ & $3.348,00$ & $-1.890,5$ \\
\hline Módulos con exceso de saberes & 19 & $3.521,92$ & $2.133,00$ & $1.388,9$ \\
\hline Total & $\mathbf{6 2}$ & $\mathbf{7 . 8 6 7 , 9 2}$ & $\mathbf{8 . 3 7 0 , 0 0}$ & $\mathbf{- 5 0 2 , 1}$ \\
\hline
\end{tabular}




\subsubsection{Comparación por competencias}

Al analizar las doce competencias a nivel de carga académica estimada podemos apreciar que existe una gran disparidad entre las competencias con el mismo nivel de logro; por ejemplo, las competencias de nivel de logro avanzado varían su número de horas de carga entre 362.75 y 1943.92 horas con una media de 670.82 horas y una desviación estándar de 625.90 horas. Mientras que las competencias de nivel de logro intermedio varían entre 165.33 horas y 1.150 .67 horas con una media de 640.5 horas y una desviación estándar 373.47 horas. Se puede apreciar una vez realizado el análisis que la diferencia entre ambos niveles de logro en promedio es demasiado baja.

Tabla 8. Resultados del análisis realizado por competencias

\begin{tabular}{|c|c|c|}
\hline Competencia & Nivel de logro & $\boldsymbol{T} \boldsymbol{M C}_{\boldsymbol{i}}$ \\
\hline 1 & Avanzado & 461,67 \\
\hline 2 & Intermedio & 331,33 \\
\hline 3 & Avanzado & 371,25 \\
\hline 4 & Intermedio & 916,50 \\
\hline 5 & Avanzado & 495,00 \\
\hline 6 & Avanzado & $1.943,92$ \\
\hline 7 & Intermedio & 165,33 \\
\hline 8 & Intermedio & 780,25 \\
\hline 9 & Avanzado & 390,33 \\
\hline 10 & Avanzado & 362,75 \\
\hline 11 & Intermedio & $1.150,67$ \\
\hline 12 & Intermedio & 498,92 \\
\hline Total & & $\mathbf{7 . 8 6 7 , 9 2}$ \\
\hline
\end{tabular}

\subsubsection{Comparación por semestre}

Al realizar el análisis desde el punto de vista de los semestres, se puede apreciar una gran disparidad entre la declaración de sistema de créditos por semestre y la valorización obtenida con el modelo propuesto. Desde el semestre dos al cinco se presenta una sub-valoración de la carga académica que alcanza un $43 \%$ en el semestre dos. Mientras que desde el semestre 6 al semestre 11 fueron sobrevaloradas; por ejemplo, el semestre 11 cuenta con una carga estimada de 40.83 horas, pero según la declaración del módulo, este requiere de 432 horas lo que significa que solo se le asigna un 9.34\% de la carga total. 
Tabla 9. Resultados del análisis realizado por semestre

\begin{tabular}{|l|l|l|l|}
\hline Semestre & \multicolumn{1}{|c|}{$\boldsymbol{T} \boldsymbol{M} \boldsymbol{C}_{\boldsymbol{i}}$} & SCT-Chile & Diferencia \\
\hline 1 & 762,00 & 810,00 & $-48,00$ \\
\hline 2 & $1.196,50$ & 837,00 & 359,50 \\
\hline 3 & $1.182,00$ & 756,00 & 426,00 \\
\hline 4 & 982,08 & 837,00 & 145,08 \\
\hline 5 & 918,00 & 756,00 & 162,00 \\
\hline 6 & 544,00 & 810,00 & $-266,00$ \\
\hline 7 & 826,00 & 999,00 & $-173,00$ \\
\hline 8 & 726,00 & 999,00 & $-273,00$ \\
\hline 9 & 354,33 & 594,00 & $-239,67$ \\
\hline 10 & 336,17 & 540,00 & $-203,83$ \\
\hline 11 & 40,83 & 432,00 & $-391,17$ \\
\hline Total & $\mathbf{7 . 8 6 7 , 9 2}$ & $\mathbf{8 . 3 7 0 , 0 0}$ & $\mathbf{- 5 0 2 , 0 8}$ \\
\hline
\end{tabular}

\section{CONCLUSIÓN Y DISCUSIÓN}

A partir de las nuevas necesidades educativas que han ido surgiendo con el trascurrir de los años, se ha debido de innovar a nivel curricular, adoptando nuevas formas de concebir la educación.

Es por estas necesidades que las aulas chilenas han debido de adoptar el modelo por competencia con la finalidad de darle un mejor estándar a la entrega de nuevos aprendizajes y de esta forma lograr obtener profesionales integrales en cada una de sus disciplinas permitiendo de esta manera que la formación superior cada día satisfaga de mejor manera las necesidades que en la actualidad se demandan.

La metodología propuesta en el presente documento describe una metodología de carácter y cuantitativa la que permite revisar de manera preliminar las competencias, los saberes y la carga académica declarada en el programa. El propósito final de esta metodología apunta a evaluar un programa basado en competencias utilizando un sistema hibrido (cuantitativo - cualitativo) para su ejecución.

La metodología se divide en cuatro etapas, las dos primeras etapas de esta metodología atienden principalmente a la de una revisión cualitativa del programa, seguidos de las otras dos etapas de carácter cuantitativas. En las primeras etapas se revisa la coherencia que existe entre la declaración de perfil de egreso y las competencias. Esta primera revisión permite más que buscar cambios radicales en el programa, busca que la institución logre ajustar ambos instrumentos. En la segunda etapa se revisa la redacción y síntesis de los saberes declarados por el programa, debido a que, según la experiencia de las autoras de este documento, las trayectorias de aprendizaje se caracterizan por tener muchos saberes con una redacción mejorable o en muchas ocasiones saberes duplicados (dos saberes que dicen los mismo, pero no son iguales).

En la tercera parte del documento se presenta la descripción del modelo matemático diseñado para la revisión de la carga académica, considerando una valorización de los saberes en base a tres variables; el LOHk la jerarquía en la taxonomía Objetivos de Aprendizaje del saber k, CDFi,i el factor de desarrollo 
de la competencia esperado en el módulo i, CDk el cual corresponde a la profundidad del contenido en el saber k.

Y en la cuarta etapa con los resultados del modelo matemático, se propone una atractiva forma de analizar la carga académica del programa en general, por semestre, por competencias y por módulo, con el propósito de identificar donde se deben realizar las mejoras a nivel de saberes y de carga académica.

$\mathrm{Al}$ analizar el perfil de egreso de una carrera de ingeniería, se pudo identificar que las competencias que se constituyen la carrera, si se encontraban estipuladas dentro del perfil de egreso de la carrera, permitiendo de esta manera que los estudiantes reciban lo acordado según lo declarado en el perfil de egreso durante su periodo académico.

Por otra parte, al realizar la revisión de los saberes de la carrera se llegó a la conclusión que muchos de ellos poseían errores de redacción, síntesis y duplicados en diversas ocasiones, por lo que se propuso realizar un análisis de estos con el objetivo central de crear una propuesta de mejora para estos.

Los primeros resultados que arrojo el estudio, presento que menos de un 50\% de los módulos de la carrera en cuestión poseía alrededor de 500 horas de déficit en cuanto a la carga asignada según las estimaciones que realizo el equipo revisor.

Por otra parte, el estudio revelo que existe una diferencia sustancial entre los primeros semestres que componen el programa en diferencia a los últimos semestres, por lo que esto genera una sobrecarga al inicio de la carrera.

Por último, se pudo observar durante la realización del estudio que las competencias que son consideradas como un nivel de logro intermedio, no presentaban mayores diferencias en la cantidad de horas asignadas en comparación con las competencias de nivel de logro avanzado por lo que no fue posible cuantificar la diferencia que se declara entre los distintos niveles de las competencias. 


\section{BIBLIOGRAFÍA}

Díaz Barriga, F. y. (2006). Desarrollo del curriculo e innovacion: Modelos e investigacion en los noventa.

Fitz-Gibbon, C.T. y Morris, L.L. (1978): How to design a Program Evaluation.

Muñoz, A. \&. (2006). Proyecto Tuning en Chile: análisis de procesos de internacionalización de la educación superior.. Calidad en la Educación, (24), 249-271. doi:https://doi.org/10.31619/caledu.n24.278

Perez, G. (2012). REDHECS: Revista electrónica de Humanidades, Educación y Comunicación Social, ISSN-e 1856-9331, 169-181.

Pérez, R. P. (2011). El diseño curricular: componentes y modelos. In Diseño y desarrollo del curriculum (p. 77). Alianza.

Tardif, J. (2006). L'évaluation des compétences. Documenter le parcours de développement . Montréal : Chenelière Éducation.

Tejedor, F. J. T. (2000). El diseño y los diseños en la evaluación de programas. Revista de investigación educativa, 18(2), 319-339.

Tobón, S. (2001). Aprender a emprender. Un enfoque curricular. Medellín: Funorie.

Tobón, S. (2002). Modelo pedagógico basado en competencias. Medellín: Corporación Lasallista.

Tobón, S. (2005). Formación basada en competencias. Pensamiento complejo, diseño curricular y didáctica, 2 ed. Bogotá: ECOE Ediciones.

Tobón, S. (2006a). Las competencias en la educación superior. Políticas de calidad. Bogotá: ECOE.

Tobón, S. (2008). La formación basada en competencias en la educación superior: el enfoque complejo. 